\title{
Immune reactions to Bacteroides fragilis populations with three different types of capsule in a model of infection
}

\author{
Sheila Patrick, ${ }^{1}$ Deborah A. Lutton' and Alistair D. Crockard ${ }^{2}$ \\ Author for correspondence: Sheila Patrick. Tel: +44 1232 240503. Fax: +44 1232438181.
}

Department of Microbiology and Immunobiology, School of Clinical Medicine, Queen's University of Belfast', and Regional Immunology Laboratory², Royal Victoria Hospital Site, Grosvenor Road, Belfast BT12 6BN, UK
The survival and growth of populations of the obligately anaerobic pathogenic bacterium Bacteroides fragilis enriched for large capsules (LCs), small capsules (SCs) or an electron-dense layer (EDL; non-capsulate by light microscopy) were examined in a mouse model of infection over a minimum period of $20 \mathrm{~d}$. Chambers which allowed the influx of leukocytes, but not the efflux of bacteria, were implanted in the mouse peritoneal cavity. The LC and EDL populations consistently attained viable cell densities of the order of $10^{8}-10^{9}$ c.f.u. $\mathrm{m}^{-1}$ within $24 \mathrm{~h}$, whereas the SC population did not. However, after $3 \mathrm{~d}$, all three bacterial populations maintained total viable numbers of $10^{8}-10^{9}$ c.f.u. $\mathrm{ml}^{-1}$ within the chambers. LC expression was selected against within $24 \mathrm{~h}$ in the model, the populations becoming non-capsulate by light microscopy, whereas in the SC population expression of the SC was retained by approximately $90 \%$ of the population. The EDL population remained noncapsulate by light microscopy throughout. Lymphocytes infiltrated the chambers to an equal extent for all three $B$. fragilis populations and at approximately 1000 times higher concentration than chambers which contained only quarter-strength Ringer's solution. The presence of neutrophils within the chambers did not cause a decrease in the total viable bacterial count. Each population elicited antibodies specific for outer-membrane proteins and polysaccharide, as detected by immunoblotting, which crossreacted with the other populations. Differences were observed in the immunogenicity of the outer-membrane proteins within the three populations. Neutrophils were initially the predominant cell type in the chambers, but as the total leukocyte count increased with incubation time, neutrophils were outnumbered by other leukocytes. Flow cytometric investigations indicated that by day 7 the majority of these leukocytes were B-cells. Bearing in mind the constraints of this model system, it appears that all three populations of $B$. fragilis have the potential for in vivo growth and that each elicits an immune reaction.

Keywords: Bacteroides fragilis, polysaccharide capsules, immune response

\section{INTRODUCTION}

The obligately anaerobic bacteria of the genus Bacteroides are numerically the predominant component of the normal commensal faecal flora of the colon in all adult humans, where obligate anaerobes outnumber facultatively anaerobic bacteria by about 1000 to 1 (Willis,

Abbreviations : EDL, electron-dense layer; LC, large capsule; RS, Ringer's solution; SC, small capsule.
1991). Of the Bacteroides species in the faecal flora, Bacteroides vulgatus predominates and is estimated to account for up to $45 \%$ of the total bacteria present. Bacteroides fragilis is less prevalent and estimates of its contribution to the total bacterial faecal flora include $4 \%$ (Namavar et al., 1989) and up to $13 \%$ (Willis, 1991). However, $B$. fragilis is estimated to account for $42 \%$ of the adherent colonic mucosal flora (Namavar et al., 1989). $B$. fragilis is the most commonly encountered species in infection resulting from faecal contamination, whereas $B$. vulgatus and other related Bacteroides spp. in the ' $B$. fragilis' 
group are infrequently isolated. Infections from which $B$. fragilis has been isolated include abdominal, pelvic, perianal and vaginal abscesses (Tally \& Ho, 1987). Peritonitis, resulting from the leakage of the bowel contents into the peritoneal cavity as a result of, for example, appendicitis or bowel surgery, is a classical example of a $B$. fragilis infection. Although $B$. fragilis and related species within the ' $B$. fragilis' group are not members of the normal commensal vaginal flora, they are also responsible for up to half of the upper genital tract, pelvic and uterine infections in women (Duerden, 1991). The majority of $B$. fragilis infections are polymicrobial; for example, Escherichia coli and Streptococcus milleri are frequently isolated in association with $B$. fragilis (Willis, 1991; Patrick et al., 1995). Although the relationship between these bacterial species may be synergistic, it is generally considered that antibiotic therapy against the anaerobic component of the polymicrobial infection is essential for successful resolution (Rotstein \& Meakins, 1990).

The precise virulence determinants of $B$. fragilis remain to be defined; however, surface structures (such as capsular polysaccharides and fimbriae), the release of extracellular enzymes which degrade components of the host tissue (e.g. neuraminidase) and the release of factors which inhibit phagocytic function may all be involved (Patrick, 1993). B. fragilis expresses at least three different types of encapsulating surface structure which can be characterized by electron microscopy of ultrathin sections; namely, a large capsule (LC), a small capsule (SC) and an electrondense layer (EDL). The EDL is visible by electron microscopy but not by light microscopy. Each of these structures may be produced within a single strain. Populations bearing these can be enriched by subculture after Percoll density-gradient centrifugation separation (Patrick \& Larkin, 1993). Labelling with monoclonal antibodies specific for surface polysaccharides reveals antigenic variation within these enriched populations (Patrick \& Lutton, 1990a).

The relationship of these encapsulating structures to virulence is not clear, but it is possible that the EDL plays a role in attachment to host cells (Patrick, 1988, 1993). The presence of the LC in $B$. fragilis impedes phagocytic uptake and killing in studies of phagocytosis in vitro (Reid \& Patrick, 1984); however, populations enriched for the LC are rapidly selected against during growth in a mouse model of infection. Within $24 \mathrm{~h}$, LC-expressing cells decrease from $99 \%$ of the population to less than $30 \%$, despite the presence of neutrophils (Patrick et al., 1984; Patrick, 1988). In contrast, after $24 \mathrm{~h}$ growth in defined medium broth culture, LC-expressing cells constitute more than $90 \%$ of the total population. During continuous subculture in vitro the population gradually reverts to a mixed capsulate and non-capsulate population over a period of days (S. Patrick, unpublished data). It may be that in vivo the bacteria switch from the production of a discrete capsule structure to release of free slime. Surfaceassociated polysaccharide in the LC population and excreted polysaccharide in the EDL population have common epitopes (Lutton et al., 1991).
In the present study we determine the immune reaction to the LC, SC and EDL populations in an infection localized within chambers implanted in the mouse peritoneal cavity over a minimum period of $20 \mathrm{~d}$. The generation of immunoglobulin specific for both outer-membrane proteins and polysaccharides was demonstrated for each population. The influx of neutrophils and B-lymphocytes into the chambers was observed and not found to affect the total viable bacterial numbers.

\section{METHODS}

Bacterial strains. The strain used in this study was Bacteroides fragilis NCTC 9343 (National Collection of Type Cultures, Colindale Avenue, London).

In vitro culture methods. Bacteria were incubated in minimal defined medium (MDM) broth (van Tassell \& Wilkins, 1978). Cultures were incubated at $37^{\circ} \mathrm{C}$ in an atmosphere of $\mathrm{H}_{2} / \mathrm{N}_{2} / \mathrm{CO}_{2}(10: 80: 10$, by vol.) in an anaerobic cabinet (MK III Don Whitley Scientific). Identification was confirmed with the API20A system.

Separation and enrichment of bacterial populations. Bacterial populations were enriched for different sizes of encapsulating surface structures by Percoll (Pharmacia) discontinuous densitygradient centrifugation as previously described (Patrick \& Larkin, 1993). Populations enriched for the SC by Percoll gradient centrifugation were further enriched, in an anaerobic atmosphere, using immunomagnetic beads (Dynabead M-450 Dynal) coated with anti-mouse immunoglobulin and mouse monoclonal antibody Bf4 (Reid et al., 1987), which is specific for the SC population; this was followed by one subculture in MDM broth. The efficiency of the immunomagnetic bead enrichment method was tested by mixing LC and SC bacteria obtained from Percoll gradient separation in a ratio of $1: 1$ and enriching from this population with the coated immunomagnetic beads. Following overnight culture in broth it was estimated by light microscopy and negative staining that less than $1 \%$ of the population had LCs. The enrichment of the SC population was confirmed by flow cytometric analysis as previously described (Lutton et al., 1991).

Preparation of bacterial outer membranes and polysaccharide. Outer-membrane proteins were prepared as previously described (Patrick \& Lutton, 1990b). In brief, bacterial suspensions were rotated 'end-over-end' for $30 \mathrm{~min}$ in 3\% (w/v) $N$-lauroylsarcosinate (Sarkosyl). After centrifugation in an Eppendorf centrifuge 5414 at $9980 \mathrm{~g}$ the supernatant fraction was discarded and the pellet containing the outer membrane was retained. Polysaccharide was extracted by the proteinase $\mathrm{K}$ method (Brown et al., 1989).

SDSPAGE and immunoblotting. SDS-PAGE was performed on vertical slab gels $(8 \%, w / v)$ and immunoblotted as previously described (Lutton et al., 1991). The human serum sample was obtained from a patient who was blood-culture positive for $B$. fragilis. The sera and chamber fluids were used at a dilution of 1 in 100 .

In vivo culture of bacteria. Chambers were constructed and implanted (two per mouse) in the mouse peritoneal cavity as previously described (Patrick et al., 1984; Lutton, 1991). The chambers were sealed with $3 \mu \mathrm{m}$ pore membrane filters (Type SSWP, Millipore) which allowed entry of leukocytes but not leakage of bacteria. If filled and sealed chambers were incubated in MDM broth there was no growth in the broth. The bacterial inoculum consisted of a suspension $(0.2 \mathrm{ml})$ of 
approximately $5 \times 10^{7}$ c.f.u. $\mathrm{ml}^{-1}$ in quarter-strength Ringer's solution (RS) containing $0.5 \mathrm{~g}$ L-cysteine $\mathrm{I}^{-1}$ (the L-cysteine helps to maintain the viability of $B$. fragilis in RS). The bacterial cultures were not exposed to air at any stage in the preparation of the chambers. All manipulations, from the standardization of the bacterial suspensions by optical density reading to filling and sealing the chambers, were carried out inside an anaerobic cabinet. Chambers were transported to the operating theatre in bottles containing RS inside a sealed anaerobic jar and only removed immediately prior to implantation in the mouse. After implantation of the chambers in the mouse for $1,3,7,14,20$ or $44 \mathrm{~d}$ the mice were killed, bled from the heart and the chambers removed. After clotting, the serum was separated and stored at $-20^{\circ} \mathrm{C}$. The chamber contents $(0.2 \mathrm{ml})$ were removed and the inside of the chamber washed out vigorously with a further $1.8 \mathrm{ml}$ sterile $0.01 \mathrm{M}$ PBS $\left(0.15 \mathrm{M} \mathrm{NaCl}, 0.0075 \mathrm{M} \mathrm{Na}_{2} \mathrm{HPO}_{4}\right.$, $\left.0.0025 \mathrm{M} \mathrm{NaH}_{2} \mathrm{PO}_{4} .2 \mathrm{H}_{2} \mathrm{O}\right), \mathrm{pH} 7.4$, in $0.2 \mathrm{ml}$ amounts. The data were obtained from four to eight mice, for each sampling day, and a minimum of two independently set-up experiments, with the exception of the initially SC population on days 7,14 and 44 where two mice were examined. Each of the chambers was examined individually, except where the total leukocyte count was low when the contents of two chambers from the same mouse were pooled for examination of the leukocyte populations. Control chambers containing only RS and RS with Percoll were also examined.

Examination of chamber contents. The total viable bacterial numbers within the chambers were determined by carrying out serial dilutions in RS and plating onto lysed blood agar as previously described (Patrick et al., 1984). All manipulations were carried out inside an anaerobic cabinet. An aliquot $(0.2 \mathrm{ml})$ of the diluted chamber contents was subcultured overnight in MDM broth. Eosin/carbol fuchsin capsule smears of bacteria, taken directly from the chambers and after overnight subculture, were examined by light microscopy (Patrick \& Larkin, 1993). The SC expression was also examined by flow cytometric analysis (Lutton et al., 1991).

Heparin $(50 \mu \mathrm{l})$ was added to $1.5 \mathrm{ml}$ of the diluted chamber contents in PBS which were incubated at $37{ }^{\circ} \mathrm{C}$ for $30 \mathrm{~min}$ to remove non-specific surface immunoglobulin from the leukocytes. The chamber contents were then centrifuged at $330 \mathrm{~g}$ for $5 \mathrm{~min}$ in an MSE Mistral 3000i and the supernatant fraction (i.e. chamber fluid) removed and stored at $-20^{\circ} \mathrm{C}$. The cell pellet was washed twice by centrifugation and resuspension in PBS containing foetal calf serum $(0.1 \%, \mathrm{v} / \mathrm{v} ; \mathrm{FCS})$. The total viable leukocyte count was determined by microscopy using a haemocytometer counting chamber with the cells suspended in trypan blue $(0.08 \%, \mathrm{w} / \mathrm{v})$. For differential staining the cells were resuspended at a concentration of $1 \times 10^{6}$ cells ml m RPMI1640 medium (Gibco) containing FCS (5\%,v/v) and centrifuged in a cytospin (Shandon Elliot) at 400 r.p.m. for $10 \mathrm{~min}$. The slides were air-dried and either stored at room temperature prior to esterase staining or at $-20^{\circ} \mathrm{C}$.

Identification of leukocyte populations. Identification of chamber-infiltrating leukocytes was carried out by morphological, cytochemical and flow cytometric techniques. Preparations from normal mouse peripheral blood and peritoneal washes were used as positive controls for the staining procedures. Morphological analyses were performed on Diff-Quik (Merz-Dade) or haematoxylin-stained preparations. Monocytes/macrophages were identified following $\alpha$-naphthol staining of leukocyte preparations ( $\mathrm{Li}$ et al., 1973).

B-lymphocytes were identified by flow cytometric analyses of cell suspensions which had been labelled with the mouse Blymphocyte marker anti-mouse Ly-5 (B220)-fluorescein-conjugated antibody (Coulter Immunology) as follows. Hepar- inized chamber contents were incubated at $37^{\circ} \mathrm{C}$ for $30 \mathrm{~min}$ to remove non-specific surface immunoglobulin, then washed twice by centrifugation in PBS containing bovine serum albumin $(2 \%, \mathrm{w} / \mathrm{v})$ and incubated with the B-cell marker for $30 \mathrm{~min}$ at $4{ }^{\circ} \mathrm{C}$ in the dark. The samples were then washed twice as before and finally suspended in PBS containing paraformaldehyde $(1 \%, v / v)$ prior to flow cytometric analysis. Heparinized mouse peripheral blood was labelled as above, except that the red blood cells were lysed with Immuno-lyse reagent (Coulter Immunology). Lysis was stopped by the addition of Immuno-lyse fixative (Coulter Immunology). The leukocytes were then washed twice as above and resuspended in PBS/paraformaldehyde. An irrelevant fluorescein-conjugated antibody, anti-human CD4 (Becton Dickinson), was used as a negative control.

Analyses were carried out with an EPICS 5 flow cytometer (Coulter Electronics) equipped with a $5 \mathrm{~W}$ argon laser and tuned to $488 \mathrm{~nm}$ and operating at a power output of $300 \mathrm{~mW}$ at the following setting: forward angle light scatter (FALS) gain, $10 ; 90^{\circ}$ light scatter (LS) detector, $420 \mathrm{~V}$; green fluorescence detector, $1300 \mathrm{~V}$. The lymphocyte population was identified on the basis of size by FALS and $90^{\circ}$ LS signals and gated appropriately. Single parameter log integral green fluorescence signals were obtained from the gated population. Five thousand cells were counted in each sample and the percentage of positively staining cells was obtained by subtraction of negative control histograms from test histograms using the instrument's Immuno-programme.

Statistics. The error bars on the graphs represent the confidence limits at $5 \%$ probability $\left(\operatorname{sigma}_{n} . t\right)$ where $n$ is a minimum of four.

\section{RESULTS}

\section{Bacterial viability and leukocyte analyses}

Populations of $B$. fragilis initially enriched for the LC, SC and EDL grew in the chambers and attained viable cell densities of over $10^{9}$ c.f.u. $\mathrm{ml}^{-1}$ (Fig. 1); however, growth of SC-enriched populations was more erratic during the

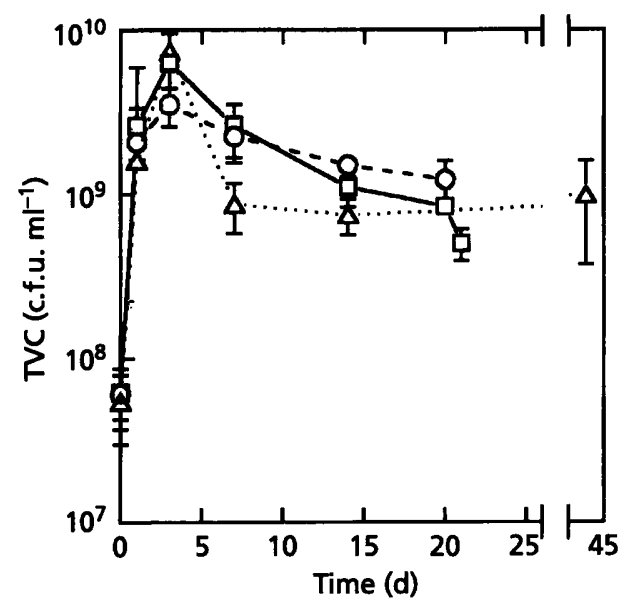

Fig. 1. Total viable bacterial counts (TVC) of B. fragilis populations enriched for the LC $\left(--\mathrm{O}_{--)}\right.$, SC $(\cdots \Delta \cdots)$ and EDL $(-\square-)$ during incubation in implanted chambers. Error bars represent confidence limits at $5 \%$ probability. 


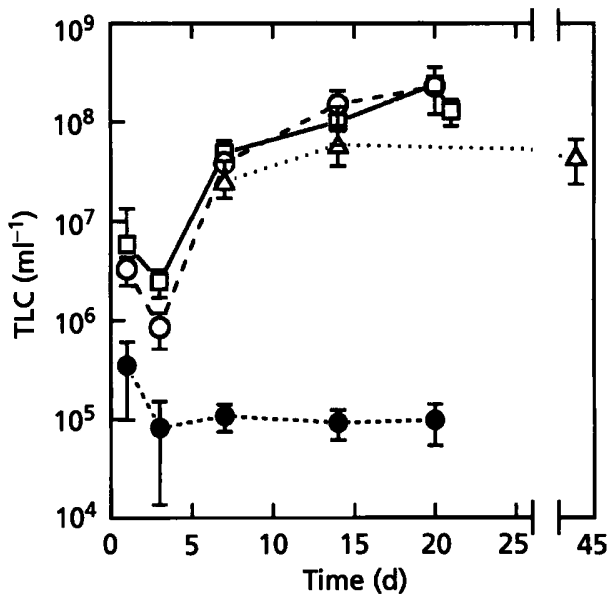

Fig. 2. Total viable leukocyte counts (TLC) in chambers containing only quarter-strength RS with $0.5 \mathrm{~g}$ L-cysteine $\mathrm{I}^{-1}$ $(----)$, or B. fragilis enriched for the LC $\left(-\mathrm{O}_{--)}\right.$, SC $(\cdots \Delta \cdots)$ and EDL (- - ) during incubation in vivo. Data are not available for the SC population on days 1 and 3. Error bars represent confidence limits at $5 \%$ probability.

first day of incubation. Out of a total of 10 mice examined $24 \mathrm{~h}$ after chamber implantation, no viable bacteria were detected in both chambers from three of the mice and the total viable bacterial count was less than or equivalent to the starting inoculum in both chambers removed from three other mice. The remainder attained total viable bacterial numbers of greater than $10^{9}$ c.f.u. $\mathrm{ml}^{-1}$. After $3 \mathrm{~d}$ incubation, the numbers of viable SC population bacteria in all the mice examined were similar to those of the LCand EDL-enriched populations. In chambers containing either the LC or EDL populations total viable bacterial counts of between $10^{8}$ and $10^{9}$ c.f.u. $\mathrm{ml}^{-1}$ were obtained consistently (Fig. 1). Host material, probably fibrin, gradually built up on the external surface of the chamber membrane filters and by day 7 the chambers were fully encased. The chamber contents also became progressively more viscous during the incubation period. Whether this was due to host- or bacteria-derived material is not known. Observation of the filters revealed that the membrane filters of control chambers without bacteria did not have the same level of deposition of host material, although they did become encased in a thin film, and their contents were less viscous.

The LC-enriched population changed from $99 \%$ LC to $97-98 \%$ non-capsulate by light microscopy within $24 \mathrm{~h}$ and remained at this level for the $20 \mathrm{~d}$ period. This decrease in LC-expressing bacteria was evident on direct examination of the chamber contents as well as after one overnight subculture of the chamber contents in MDM broth. The change was therefore not due to environmental modulation. This decrease in the number of LC-expressing bacteria does not occur on subculture directly from the Percoll gradient into MDM broth where $95-100 \%$ of the bacteria continue to express the LC. In populations enriched for the SC, an average of $91 \%$

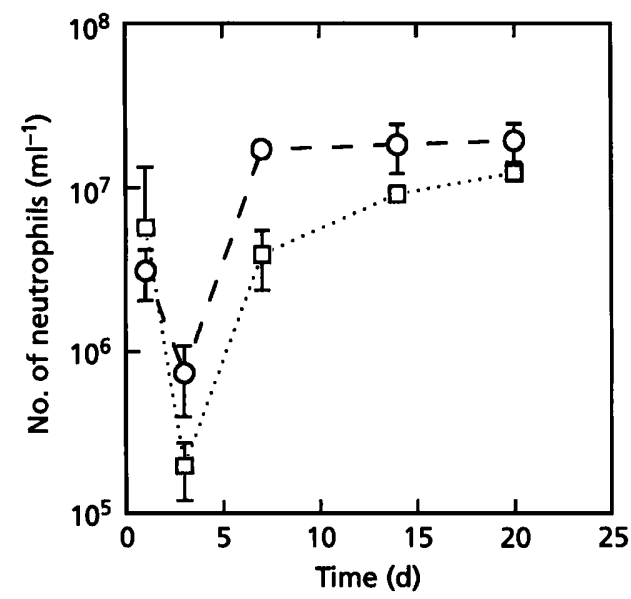

Fig. 3. Numbers of neutrophils in chambers containing $B$. fragilis populations enriched for the LC $(O)$ and EDL $(\square)$. Data were obtained from differential microscopical counts and calculated from the total leukocyte count. Data are not available for the SC population. Error bars represent confidence limits at $5 \%$ probability.

(range $80-96 \%$ ) of bacteria were estimated to express the SC by light microscopy and an average of $96 \%$ (range 90-99\%) by flow cytometric analysis using monoclonal antibody Bf4, throughout the $44 \mathrm{~d}$ incubation period. The rest of the population was non-capsulate by light microscopy, with the exception of day 7 , where $1.5 \%$ of the population expressed the LC. The EDL population remained non-capsulate by light microscopy when examined directly upon removal from the chamber and also after one subculture of the chamber contents into MDM broth.

The total leukocyte count in the chambers increased progressively with the duration of implantation in the mouse after an initial decrease between day 1 and day 3, and was in excess of that observed in control chambers containing only diluent (quarter-strength RS with $0.5 \mathrm{~g} \mathrm{~L}-$ cysteine $\mathrm{l}^{-1}$ ) (Fig. 2). To determine if any residual Percoll in the bacterial inocula had an effect, an $80 \%$ suspension of Percoll, diluted 1 in 500 in RS, was incubated in the chamber model for 3 and $21 \mathrm{~d}$. This resulted in similar leukocyte infiltration to that observed with chambers containing only RS.

Differential counts performed on Diff-Quik-stained cytospin preparations indicated that neutrophils were the predominant infiltrating cell at day $1(97 \%$ and $94 \%$ for chambers containing the EDL and LC populations, respectively). However, by day 14 , against a background of increasing total leukocyte count (Fig. 2), neutrophils comprised only $9 \%$ (EDL chambers) and $12 \%$ (LC chambers) of the total leukocyte count. The numbers of neutrophils in the chambers are illustrated in Fig. 3. Neutrophils containing phagocytosed bacteria were observed by electron microscopy (not illustrated). Classification of the other leukocytes by microscopy proved difficult due to the distorted morphology of the cells. It is 

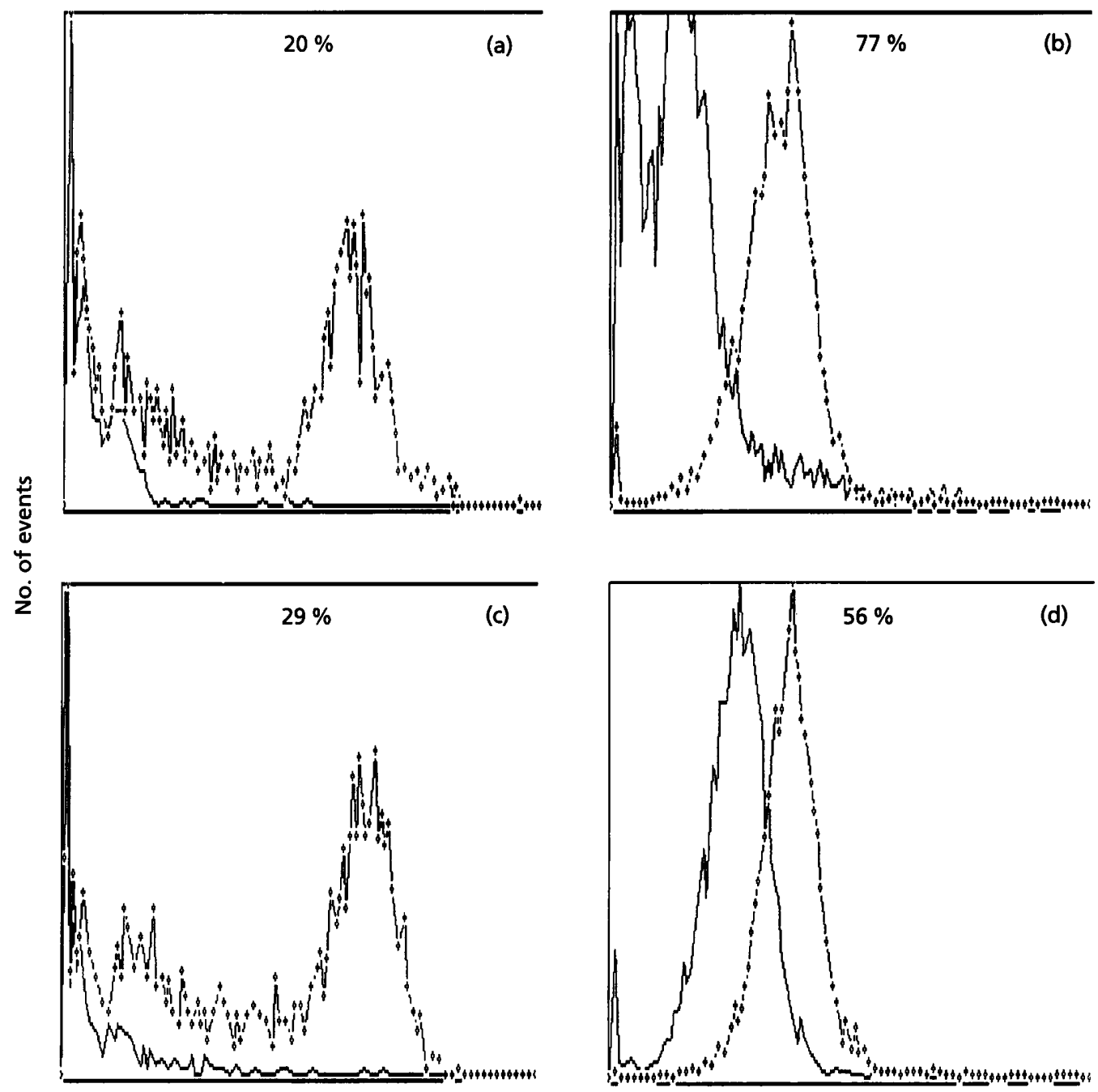

Fluorescence intensity

Fig. 4. Representative flow cytometric profiles of Ly-5 (B220) expression by lymphocytes from mice with implanted chambers containing $B$. fragilis enriched for the EDL population after $\mathbf{7 d}(a, b)$ and $21 d(c, d)$ incubation. (a, $c)$ Peripheral blood; $(b, d)$ chamber contents; dotted line, Ly-5 (B220) positive; solid line, control anti-human T-lymphocyte CD4; X-axis, fluorescence intensity channel number $0-255 ; Y$-axis, number of events scale $0-50$. The means of the percentages of Ly-5 (B220)-positive leukocytes are indicated on each graph.

possible that the altered shape of the cells present in the chambers occurred as a result of cellular activation. Cytochemical staining for $\alpha$-naphthol acetate esterase activity was observed with circulating monocytes and peritoneal macrophages, but not with mononuclear cells in the chambers containing B. fragilis or diluent.

Experiments with the EDL population indicated that leukocytes which entered the chamber after the initial influx of neutrophils were B-lymphocytes. Flow cytometric profiles (Fig. 4) of Ly-5 (B220)-labelled cells indicated that on day 7 approximately $75 \%$ of the mononuclear cells were positive. Analysis of leukocytes from day 21 samples, however, revealed a high level of labelling of chamber leukocytes with the control antibody. As the proportion of the cells positive for Ly-5 (B220) is calculated relative to labelling with the control antibody, this resulted in an apparent decrease in the numbers of Ly-5 (B220)-positive cells.

\section{Immunoblotting}

Sera taken from the mice in which chambers had been implanted were reacted with electroblots of proteinase $\mathrm{K}$ (polysaccharide) and Sarkosyl (outer membrane) extracts of each of the LC, SC and EDL populations and detected with alkaline-phosphatase-conjugated anti-mouse IgG (heavy and light chains). Immunoblotting showed a progressive increase in labelling during the time of incubation in the mice. Each of the three populations elicited antibodies reactive with the other populations. Protein and polysaccharide antigens each elicited specific antibody. Selected immunoblots illustrating the reactivity with Sarkosyl extracts are illustrated in Fig. 5. A number 


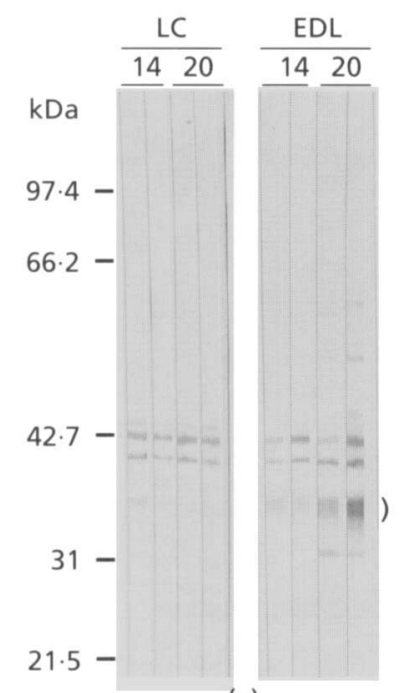

(a)

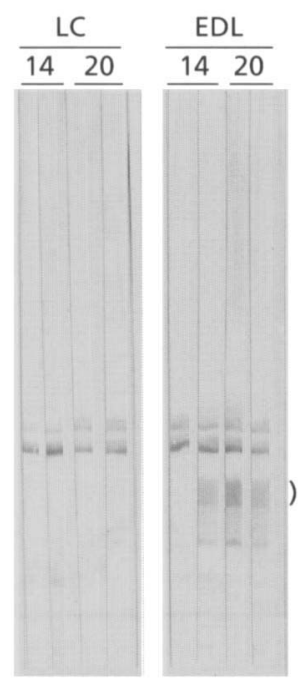

(b)

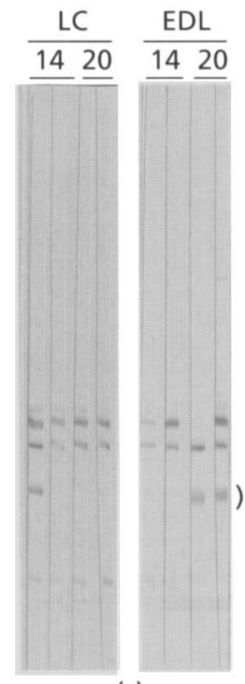

(c)
Fig. 5. Representative immunoblots from polyacrylamide gels of Sarkosyl extracts of in-vitro-grown B. fragilis enriched for the LC (a), SC (b) and EDL (c) populations probed with sera from mice in which chambers containing either the LC- or EDL-enriched populations had been implanted. The numbers represent the duration in days of implantation of chambers in the mouse. Note broad band antigens (brackets) which are not reactive with the LC sera. The reaction of sera from mice with SCcontaining chambers is not illustrated. Reaction with sera from mice with chambers containing only quarter-strength RS with $0.5 \mathrm{~g}$ cysteine $\mathrm{I}^{-1}$ did not produce a pattern of bands. Molecular mass markers are indicated on the left.

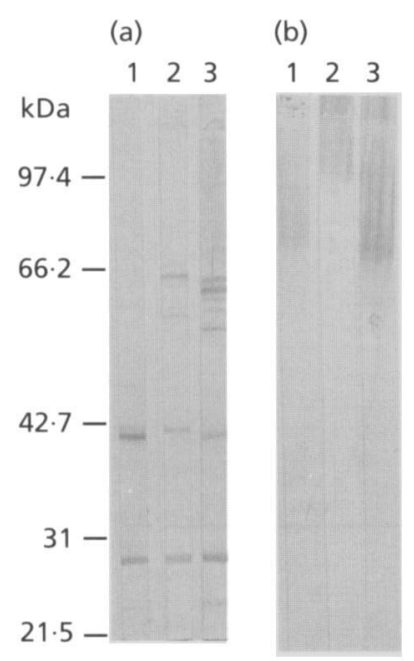

Fig. 6. Immunoblot from polyacrylamide gels of Sarkosyl (a) and proteinase $K(b)$ extracts from in-vitro-grown $B$. fragilis enriched for the LC (lane 1), SC (lane 2) and EDL (lane 3) populations probed with a human serum sample from a patient whose blood was culture positive for $B$. fragilis and anti-human IgG alkaline phosphatase conjugate. Molecular mass markers are indicated on the left.

of antigens present in the LC, EDL (Fig. 5) and SC (not illustrated) Sarkosyl extracts in the $31-40 \mathrm{kDa}$ region did not react with the sera from mice implanted with the LC population. In the proteinase $\mathrm{K}$ extracts a broad diffuse band in the high molecular mass region of the blot, with an associated fine ladder pattern was observed. This is characteristic of high molecular mass polysaccharide and similar to the pattern previously observed with monoclonal antibody labelling (Reid et al., 1987; Lutton et al., 1991). There was no labelling of the polysaccharide recognized by QUBf5 (Lutton et al., 1991), which has a ladder band-pattern similar to that of the $\mathrm{O}$-antigen of other bacteria. The reactivity of antibodies in chamber fluids, obtained after removal of the bacteria and leuko- cytes by centrifugation, with Sarkosyl-extracted outermembrane proteins was also examined. Specific antibody was detected later than in the mouse sera and was of a lower titre. The reactivity of one human serum sample from a patient from whose blood $B$. fragilis had been isolated (Fig. 6) was also compared. The human serum reacted with outer-membrane proteins and polysaccharides from the LC, SC and EDL populations.

\section{DISCUSSION}

The growth of the LC- and EDL-enriched populations within the chambers was consistent and reproducible. The exception was the SC population, which on occasion failed to grow or did not reach high viable numbers within the first $24 \mathrm{~h}$ of implantation. However, where the $\mathrm{SC}$ population did survive, by $3 \mathrm{~d}$ the viable numbers were equivalent to those of the other populations and were maintained at that level for up to $44 \mathrm{~d}$. It is possible that the SC population has to adapt in some way for growth in the peritoneal environment, or that some selection is taking place within the population which is not necessary for the initial survival of the LC and EDL populations. The chamber model did not require the addition of, for example, E. coli or adjuvant for bacterial growth. $B$. fragilis cells for implantation were suspended in quarter-strength RS with L-cysteine $\left(0.5 \mathrm{~g} \mathrm{l}^{-1}\right)$ and not exposed to oxidizing conditions, which reduce total viable numbers. It may be that the dependence of $B$. fragilis on $E$. coli for survival within the peritoneal cavity in a fibrin clot model of infection (Verweij et al., 1991) relates to the metabolic activity of the $E$. coli which keeps the surrounding environment suitably reduced, thus enabling $B$. fragilis to maintain viability. This eventually results in an environment which is sufficiently reduced to allow multiplication of the $B$. fragilis. Synergy between $B$. fragilis and E. coli, in the initial stages of infection, may be related to redox potential.

Monitoring of the cellular response to chambers containing bacteria indicated that the host leukocytes entered 
the chambers in quantity and that there was no apparent difference between the LC, SC and EDL populations in terms of the numbers of leukocytes which entered the chambers. In concordance with the findings of Verweij et al. (1991), neutrophils were readily identified within the chambers as the initially predominant cell type. In their studies of mixed infections of $E$. coli and $B$. fragilis strain BE1, neutrophils were the predominant cell type $2 \mathrm{~h}$ after implantation of a fibrin clot in the rat peritoneal cavity. In the present study, labelling with the Ly-5 (B220) mouse Blymphocyte marker suggests that B-lymphocytes become the predominant cell type within the chamber by day 7 , although after $21 \mathrm{~d}$ incubation, non-specific labelling with the control antibody made B-lymphocyte quantification difficult. The total viable leukocyte count, however, remained high. The non-specific labelling may be due to immunoglobulin associated with the leukocyte surface membrane or be a consequence of alteration of the surface membrane receptors resulting from cellular activation. Interestingly, the leukocytes apparently remain viable at a redox potential sufficiently low to support the survival and multiplication of $B$. fragilis.

Immunoglobulins specific for protein and polysaccharide antigens were detectable in blood sera, which indicated that each of the LC, EDL and SC populations elicited immunoglobulin which cross-reacted with the other populations. It therefore appears that sufficient bacterial antigens leak from the chambers to elicit antibody production in secondary lymphoid organs and tissues. Immunoglobulin, of a lower titre, was also detectable in the chamber fluids. This may reflect either diffusion of antibody molecules back into the chambers or generation of antibody by the infiltrating B-lymphocytes. The immunoblots showed differences between the immunoglobulin elicited by the LC population when compared with the SC and EDL populations. Certain epitopes in the $31-40 \mathrm{kDa}$ region, although present in the LC population, did not elicit specific immunoglobulin (Fig. 5), whereas the same epitopes in the SC and EDL populations did. It would be of interest to examine the reactivity of these sera against bacterial populations grown under iron-restricted conditions to determine if there was immunoglobulin specific for the $44 \mathrm{kDa}$ iron-repressible outer-membrane protein (Otto et al., 1991). Examination of the single human serum sample indicated that there were immunoglobulins specific for proteins and high molecular mass polysaccharide epitopes in LC, SC and EDL populations. Examination of polysaccharide expression in bacteria present in pus samples from a variety of different body sites by use of monoclonal antibodies indicates that epitopes associated with the LC, SC and EDL may each be present (Patrick et al., 1995).

In spite of the immune reaction, in terms of immunoglobulin production, the influx of leukocytes into the chambers and the presence of quantities of phagocytosed bacteria inside neutrophils, the number of viable bacteria remains high. How this relates to the virulence determinants of the bacteria and how much the activities of the leukocytes are impaired are as yet unresolved questions. Studies on neutrophils removed from B. fragilis-induced abscesses indicate that, although the $B$. fragilis cells inside the neutrophils are not killed, the neutrophils are still functional (Finlay-Jones et al., 1991).

This model of infection allows both the growth of bacteria and the immune response to be monitored over a period of weeks. As the bacteria cannot escape from the chamber, but host cells and fluids can enter, this model is a compromise between the open-ended chamber models, from which bacteria may translocate, and the enclosed chambers, which do not allow interaction of the bacteria with host cells (Genco \& Arko, 1994). The model is useful, not only because it yields data about fundamental aspects of the immune response to bacterial infection, but it also provides a model for prolonged infection. Whether or not an infection is established in the initial instance may be dependent on bacteria-immune-system interactions in the first few hours after contamination by the bacteria. This has obvious implications for the prevention of postoperative infections (Verweij et al., 1991). However, an abscess, once established, may persist for long periods and infections may recur at the same site. For example, $B$. fragilis has been isolated from persistent pilonidal and perineal abscesses and persistent and recurrent sepsis in the peritoneal cavity (Rotstein \& Meakins, 1990). As the bacterial population is limited to the confines of the chamber, any changes in the bacterial population can be related directly to the growth and survival of the bacterial inoculum and not to leakage of the bacteria.

The relationship between this chamber model and biofilm formation on prosthetic devices and implants is also worth consideration, particularly with a view to using the model for the determination of antibiotic activity in vivo. The chamber contents are highly viscous and packed with bacteria and leukocytes. The viscosity of the chamber contents may be due to fibrin matrix, as occurs in abscesses (Rotstein, 1992), other host components, bacterial polysaccharide or a mixture of components.

In conclusion, the results of this study indicate that, within the confines of this model, the LC, SC and EDL populations each have the potential for growth in vivo, and each elicits an immune reaction. This model therefore allows the detailed study of the interactions of $B$. fragilis with the immune system.

\section{ACKNOWLEDGEMENTS}

The authors thank Dr T. A. McNeill for his support and encouragement. The work and D. A. Lutton were funded by the Medical Research Council UK

\section{REFERENCES}

Brown, R., Hornett, K. E. \& Poxton, I. R. (1989). Immunochemistry of the cell surfaces of Bacteroides bivius and Bacteroides disiens. $J$ Med Microbiol 28, 267-273.

Duerden, B. I. (1991). Anaerobes in genitourinary infections. In Anaerobes in Human Disease, pp. 224-244. Edited by B. I. Duerden \& B. S. Drasar. London: Edward Arnold. 
Finlay-Jones, J. J., Hart, P. H., Spencer, L. K., Nulsen, M. F., Kenny, P. A. \& McDonald, P. J. (1991). Bacterial killing in vitro by abscessderived neutrophils. $J$ Med Microbiol 34, 73-81.

Genco, C. A. \& Arko, R. J. (1994). Animal chamber models for study of host-parasite interactions. Methods Enzymol 235, 120-140.

Li, C. Y., Lam, K. W. \& Yam, L. T. (1973). Esterases in human leukocytes. J Histochem Cytochem 21, 1-12.

Lutton, D. A. (1991). Variability of surface structure expression in Bacteroides fragilis. PhD thesis, The Queen's University of Belfast.

Lutton, D. A., Patrick, S., Crockard, A. D., Stewart, L. D., Larkin, M. J., Dermott, E. \& McNeill, T. A. (1991). Flow cytometric analysis of within-strain variation in polysaccharide expression by Bacteroides fragilis by use of murine monoclonal antibodies. J Med Microbiol 35, 229-237.

Namavar, F., Theunissen, E. B., Verweij-van Vught, A. M., Peerbooms, P. G., Bal, M., Hoitsma, H. F. \& MacLaren, D. M. (1989). Epidemiology of the Bacteroides fragilis group in the colonic flora of patients with colonic cancer. $J$ Med Microbiol 29, 171-176.

Otto, B. R., Verweij, W. R., Sparrius, M., Verweij-van Vught, A. M. J. J., Nord, C. E. \& Maclaren, D. M. (1991). Human immune-response to an iron-repressible outer-membrane protein. Infect Immun 59, 2999-3003.

Patrick, S. (1988). Phagocytosis of Bacteroides fragilis in vitro. In Anaerobes Today, pp. 31-41. Edited by J. M. Hardie \& S. P. Boriello. Chichester: J. Wiley.

Patrick, S. (1993). The virulence of Bacteroides fragilis. Rev Med Microbiol 4, 40-49.

Patrick, S. \& Larkin, M. J. (1993). Attachment in disease. In Microbial Biofilms: Formation and Control, pp. 109-131. Edited by S. P. Denyer, S. P. Gorman \& M. Sussman. Oxford: Blackwell Scientific.

Patrick, S. \& Lutton, D. A. (1990a). Bacteroides fragilis surface structure expression in relation to virulence. Med Mal Infect (bors serie) 20, 19-25.
Patrick, S. \& Lutton, D. A. (1990b). Outer membrane proteins of Bacteroides fragilis grown in vivo. FEMS Microbiol Lett 71, 1-4.

Patrick, S., Reid, J. H. \& Larkin, M. J. (1984). The growth and survival of capsulate and non-capsulate Bacteroides fragilis in vivo and in vitro. $J$ Med Microbiol 17, 237-246.

Patrick, S., Stewart, L. D., Damani, N., Wilson, K. G., Lutton, D. A., Larkin, M. J., Poxton, I. \& Brown, R. (1995). Immunological detection of Bacteroides fragilis in clinical samples. J Med Microbiol 42 (in press).

Reid, J. H. \& Patrick, S. (1984). Phagocytic and serum killing of capsulate and non-capsulate Bacteroides fragilis. J Med Microbiol 17, 247-257.

Reid, J. H., Patrick, S. \& Tabaqchali, S. (1987). Immunochemical characterization of a polysaccharide antigen of Bacteroides fragilis with an IgM monoclonal antibody. J Gen Microbiol 133, 171-179.

Rotstein, O. D. (1992). Role of fibrin deposition in the pathogenesis of intraabdominal infection. Eur J Clin Microbiol Infect Dis 11, 1064-1068.

Rotstein, O. D. \& Meakins, J. L. (1990). Diagnostic and therapeutic challenges of intraabdominal infections. World J Surg 14, 159-166.

Tally, F. P. \& Ho, J. L. (1987). Management of patients with intraabdominal infection due to colonic perforation. Curr Clin Top Infect Dis 8, 266-295.

van Tassell, R. L. \& Wilkins, T. D. (1978). Isolation of auxotrophs of Bacteroides fragilis. Can J Microbiol 24, 1619-1621.

Verweij, W. R., Namavar, F., Schouten, W. F. \& MacLaren, D. M. (1991). Early events after intra-abdominal infection with Bacteroides fragilis and Escherichia coli. J Med Microbiol 35, 18-22.

Willis, A. T. (1991). Abdominal sepsis. In Anaerobes in Human Disease, pp. 197-223. Edited by B. I. Duerden \& B. S. Drasar. London: Edward Arnold.

Received 15 December 1994; revised 16 March 1995; accepted 31 March 1995. 